\title{
GALAXY HALO FORMATION IN THE ABSENCE OF VIOLENT RELAXATION AND A UNIVERSAL DENSITY PROFILE OF THE HALO CENTER
}

\author{
A. N. BAUSHEV \\ DESY, D-15738 Zeuthen, Germany; baushev@gmail.com \\ AND \\ Institut für Physik und Astronomie, Universität Potsdam, D-14476 Potsdam-Golm, Germany \\ Received 2013 August 29; accepted 2014 March 10; published 2014 April 16
}

\begin{abstract}
While $N$-body simulations testify to a cuspy profile of the central region of dark matter halos, observations favor a shallow, cored density profile of the central region of at least some spiral galaxies and dwarf spheroidals. We show that a central profile, very close to the observed one, inevitably forms in the center of dark matter halos if we make a supposition about a moderate energy relaxation of the system during the halo formation. If we assume the energy exchange between dark matter particles during the halo collapse is not too intensive, the profile is universal: it depends almost not at all on the properties of the initial perturbation and is very akin, but not identical, to the Einasto profile with a small Einasto index $n \sim 0.5$. We estimate the size of the "central core" of the distribution, i.e., the extent of the very central region with a respectively gentle profile, and show that the cusp formation is unlikely, even if the dark matter is cold. The obtained profile is in good agreement with observational data for at least some types of galaxies but clearly disagrees with $N$-body simulations.
\end{abstract}

Key words: astroparticle physics - dark matter - elementary particles - large-scale structure of universe

\section{INTRODUCTION}

The problem of universe structure formation is still far from a complete solution. Since the task is essentially nonlinear, $N$ body simulation is one of the possible ways to solve it. While the results satisfactorily describe observed properties of galaxy clusters (Okabe et al. 2010), some discrepancy seems to appear when we consider galaxy halos. $N$-body simulations predict a very steep Navarro-Frenk-White-like density profile of the central region of the dark matter (DM) halos (Navarro et al. 1997). The Navarro-Frenk-White (NFW) profile behaves there as $\rho \propto r^{-1}$. Although recent simulations favor the Einasto profile (Moore et al. 1998; Gao et al. 2008; Diemand et al. 2008; Stadel et al. 2009; Navarro et al. 2010),

$$
\rho=\rho_{s} \exp \left[-2 n\left\{\left(\frac{r}{r_{s}}\right)^{\frac{1}{n}}-1\right\}\right],
$$

with a finite density in the center (Einasto 1965), the index of the profile is so high (typically, $n \simeq 5-6$ ) that it can also be considered as a cuspy one.

On the contrary, observations (de Blok et al. 2001; de Blok \& Bosma 2002; Marchesini et al. 2002; Gentile et al. 2007) show that galaxies have a core (i.e., a region with a rather shallow density profile) in the center. Chemin et al. (2011) fitted the DM density profiles of a large array of spiral galaxies by the Einasto profile, considering the Einasto index $n$ as a free parameter. They found that the Einasto profile provides a significantly better fit of observational data of the central region than either NFW or the cored pseudo-isothermal profiles. However, the Einasto index is found to be small ( $n \simeq 0.5-1)$, which actually corresponds to a cored profile and is not in agreement with the predictions from $\Lambda \mathrm{CDM}$ simulations. Dwarf galaxies also show no cusps in their center (Oh et al. 2011; Governato et al. 2012).

There are several possible ways to account for this disagreement. It can be caused by the influence of baryon matter (Pontzen \& Governato 2012). However, we may cast some suspicion on this explanation. The fraction of baryons in some dwarf galax- ies is so tiny that it can hardly influence the cusp formation (Garrison-Kimmel et al. 2013), but these objects show no cusps in their center. Meanwhile, galaxy clusters have a much larger fraction of baryons, and their density profiles are rather steep (Okabe et al. 2010). The problem of the baryon influence on the DM distribution is very complex and not yet quite clear. It lies beyond the scope of the present work, and we will not discuss it in this paper anymore, referring readers to the vast literature on this topic (see Weinberg et al. 2013; Pontzen \& Governato 2014, and references therein). The absence of the cusps could be a sign that the DM is warm and consists of light particles like sterile neutrinos. Thus the "cusp versus core" problem might shed light on the physical nature of DM. However, we should first be sure that there is no explanation for the cores in the framework of the cold dark matter (CDM) paradigm.

We will try to show in this paper that the energy evolution of the forming halo is probably the key to the problem of central cusp occurrence. Although the density shape in the center of the halo may differ from the NFW profile, we will use the NFW halo concentration $c_{\text {vir }}$ to qualitatively characterize a halo because of the popularity of the NFW profile. Let us denote by $\epsilon=v^{2} / 2+\phi$ the total energy of a unit mass of matter, where $\phi$ is the gravitational potential. It is easy to show (see the beginning of Section 2) that in the very general case the particles that later form the halo have a very narrow initial energy distribution: the initial energies of the particles differ by no more than a factor of two. When a halo collapses, remarkable inhomogeneities and caustics appear; their strong small-scale gravitational field mediates a relaxation and may significantly smear out the energy spectrum of the DM particles with respect to the initial one. The following question arises: how strong can the relaxation be? That is, can the energy evolution of the system be arbitrarily strong, or is the ratio between the final $\epsilon_{f}$ and the initial $\epsilon_{i}$ energies somehow limited for the majority of the particles?

In principle, the ratio $\epsilon_{f} / \epsilon_{i}$ could be arbitrarily large: the idea of "violent relaxation" (Lynden-Bell 1967) was the supposition that a particle completely forgets its initial state 
during relaxation and its final energy has no connection with the initial one. However, the equilibrium solution obtained by Lynden-Bell (1967) has infinite total mass, and its applicability to finite systems is debatable. The main fraction of mass of the future halo is initially concentrated in the outer layers with the highest initial $r$ (since their volume grows as $r^{2}$ ). Meanwhile, as shown by Lynden-Bell (1967), the efficiency of all relaxation processes (and therefore the alternation of the particle energy) rapidly drops with $r$ : even in that work, the outer part of the system remained unrelaxed. Moreover, simulations show that a significant part of the total mass of the halo accretes to the already collapsed halo (Wang et al. 2012). This substance has a respectively high total energy and cannot be significantly affected by violent relaxation.

Unfortunately, halo collapse is a very complex nonlinear process, and there is almost no hope of finding an exact theoretical solution to the problem. The literature offers several possible approaches to the task. Syer \& White (1998) suggested that the cuspy DM halos might arise as a consequence of hierarchical structure formation. However, this theory predicts very steep cusps $\left(\rho \propto r^{-2}\right)$ in the case of the Harrison-Zel'dovich spectrum of initial perturbations. This cusp shape is hardly comparable with modern observations and simulations, while the Planck Collaboration et al. (2013) firmly confirm the Harrison-Zel'dovich spectrum. An another approach is to introduce Boltzmann's $H$ function (Tremaine et al. 1986; Stiavelli \& Bertin 1987), which is actually the entropy of the system. The system cannot reach the real maximum entropy, which is an isothermal sphere, but the authors suggest that the increasing of the $H$ function defines the direction of the system evolution. However, Pontzen \& Governato (2013) correctly pointed to the possible difficulties of this approach. Violent relaxation (however violent it is) is actually collisionless relaxation through the common field. This process is, in principle, reversible, and entropy does not grow in it (Landau \& Lifshitz 1980; Pontzen \& Governato 2013). A good illustration for this statement is the existence of stationary solutions, like Osipkov (1979) and Jaffe (1983). These systems are very far from the entropy maximum; in particular, the local velocity distribution there is not Maxwellian. However, they cannot relax anymore since their gravitational field is already stationary. The only process that guarantees entropy growth is the particle collisions. The characteristic time of the collisional relaxation is (Binney \& Tremaine 2008, Equation (1.38))

$$
\tau_{r}=\frac{N(r)}{8 \ln \Lambda} \tau_{d},
$$

where $v, N(r), \ln \Lambda$, and $\tau_{d}=r / v$ are the "characteristic" particle velocity, the number of particles inside radius $r$, the Coulomb logarithm, and the dynamical time of the system at $r$, respectively. The dynamical time of the galaxies is typically only a few orders of magnitude smaller than the age of the universe. Since a galactic halo may contain $\sim 10^{65}$ particles (accepting the DM particle mass of $200 \mathrm{GeV}$ ), the relaxation time enormously exceeds the age of the galaxies. So entropy growth can be completely negligible at the cosmological time. Moreover, the system can hardly be ergodic in this case, which is necessary to use the statistical methods (Pontzen \& Governato 2013). Surely, the statistically stable state is the isothermal sphere, i.e., it is cuspy. However, the applicability of the essentially statistical approach to a system that has existed for only a tiny fraction of the true relaxation time is debatable.
$N$-body simulations give a more direct way to investigate the halo relaxation. The idea of the method is to substitute real tiny DM particles by massive test bodies with smoothed Newtonian potential. Recent $N$-body simulations (Diemand et al. 2005, 2007; Diemand \& Kuhlen 2008) indicate that the supposition of violent relaxation is not true: the energies of the majority of the particles do not change completely and correlate with the initial ones. Nevertheless, the relaxation is rather strong, and the halo profiles are cuspy.

The method has a weak point, however: the number of test bodies is small compared with real DM particles, and the influence of the unphysical test body collisions should be carefully avoided. According to Equation (2), the relaxation time is proportional to $N(r)$. The closer we get to the halo center, the smaller the number of bodies $N(r)$ is. At some radius $r_{\text {conv }}$ the ratio of the simulation time $t_{0}$ to the relaxation time $\tau_{r}$ may become so large that the collisions become important. So the simulations are still reliable outside the convergence radius $r_{\text {conv }}$, but the density profile is already corrupted by the unphysical test body collisions inside $r_{\text {conv }}$.

As we have already mentioned, the DM in simulations rather rapidly forms stationary halos with an almost universal density profile. The profile is close to the NFW, being cuspy $\left(\rho \propto r^{-\gamma}\right.$, $\gamma \simeq 1$ ) or very steep in the center. The persistent appearance of the cusp and its stability with respect to parameter variations of $\mathrm{N}$-body codes are usually considered proof of its physical nature and independence of the collisions. The method commonly used (Navarro et al. 2010) to assure the negligibility of the collisions is to find the moment when the cusp starts to smear out, which is believed to be the first sign of the influence of collision (Power et al. 2003). However, the cusp turns out to be surprisingly stable. Power et al. (2003) found that the smearing does not appear at least up to $t=1.7 \tau_{r}$ and probably much longer. Indeed, Hayashi et al. (2003) and Klypin et al. (2013) observed that the cusp was stabile even at tens of relaxation times and only then smeared out. The negligibility of the test body collisions at $t \sim 30 \tau_{r}$ seems suspicious. In order to test the influence of the particle encounters analytically the Fokker-Planck equation was used (Evans \& Collett 1997; Baushev 2013c). It turns out that the density distribution close to NFW $\left(\rho \propto r^{-\gamma}, \gamma \simeq 1\right)$ is an attractor solution for the kinetic equation, taking into account close encounters. The Fokker-Planck diffusion occurring as a result of the collisions transforms any initial density distribution shallower than $\rho \propto r^{-1}$ into a NFW-like profile $(\gamma \simeq 1)$ at a time $t<\tau_{r}$. After being formed, the cuspy profile should survive for a much longer time interval since the collision effects are self-compensated to a first approximation in this case. Moreover, since the shape of the attractor solution is not sensitive to the simulation parameters, the cusp should also be insensitive to them. At tens of relaxation times $\tau_{r}$ the NFW-like profile should be smeared out by the "thermal conductivity," which is a second-order effect of particle collisions (Quinlan 1996; Baushev 2013c).

These results perfectly describe the simulation behavior. The cusp forms at $t<\tau_{r}$, survives for several or even many relaxation times, and then smears out in tens of relaxation times. However, since the test body collisions are unphysical and correspond to nothing real, the cusp formation by the Fokker-Planck diffusion is a purely numerical effect. As we can see, the collisions of real DM particles are completely ineffective. Thus, the commonly used criterion of the convergence radius $r_{\text {conv }}$ of simulations with $t<1.7 \tau_{r}$ (Power et al. 2003) seems excessively optimistic. The fact that the cusp is stable and insensitive to the 
simulation parameters is not enough of a reason to state that the profile is not affected by the unphysical collisions. The only reliable criterion is $t \ll \tau_{r}$. However, it requires many more test bodies inside $r_{\text {conv }}$ and so increases the convergence radius by several orders of magnitude. This can be of vital importance for the cusp versus core problem. Actually, the disagreement between the simulations and observations only occurs quite close (a few percent of $R_{\mathrm{vir}}$ ) to the halo center. If we use the more reliable criterion $t \ll \tau_{r}$, the contradictions may disappear.

The main aim of this paper is to show that a cored profile, which is very similar to that observed in the center of galaxies, inevitably forms in the center of DM halos if we make only the supposition that the energy relaxation of galactic DM halos was moderate in the following sense.

Principle assumption. For the majority of the particles, the final energy in the halo formed $\epsilon_{f}$ differs by no more than $c_{\text {vir }} / 3$ from the initial value $\epsilon_{i}$,

$$
\frac{\epsilon_{f}}{\epsilon_{i}} \leqslant \frac{c_{\mathrm{vir}}}{3}
$$

The concentration $c_{\text {vir }}$ of the Milky Way lies between 12 and 17 (Klypin et al. 2002) and should be even higher for smaller galaxies (Navarro et al. 2010). Therefore, the moderate relaxation supposition means that the energies of the majority of the particles have not changed by more than a factor of 4-5 that of the Milky Way; for less massive halos the assumption is even softer. Of course, there are always particles whose energies have changed more than given by Equation (3); however, we suppose that their fraction is small. The condition of moderate relaxation will be specified and restated in Section 4.

We should underline that we suggest moderate relaxation for only relatively low-mass objects like halos of galaxies. Moreover, despite all the reasoning above, this is no more than a hypothesis: the assumption cannot be certainly proved, although the validity of all the results of this article depends on the feasibility of this basic supposition. However, as we will see, the fulfillment of condition (3) automatically leads to a central density profile that is in good agreement with observations. For this reason alone, it is worth considering moderate relaxation, at least as a hypothesis.

\section{ENERGY SPECTRUM}

Let us consider the initial distribution of matter. Hereafter, in this article we will consider a spherically symmetric task and neglect the presence of baryon matter. The mass and initial radius of the halo are $m$ and $\mathfrak{R}, \mathfrak{R} \simeq R_{\mathrm{vir}}$. If the DM is cold, the initial velocity of the matter may be considered to be zero without loss of generality (Gorbunov \& Rubakov 2011). (As we will see, the conclusions of this section are also valid for warm DM). To start, let us consider the case when matter is uniformly distributed inside $\mathfrak{R}(\rho=$ const, the Tolman case). Inside the sphere,

$$
\begin{gathered}
\phi(r)=\frac{G m}{\mathfrak{R}}\left(\frac{1}{2}\left(\frac{r}{\mathfrak{R}}\right)^{2}-\frac{3}{2}\right), \\
d m=3 \frac{m}{\mathfrak{R}}\left(\frac{r}{\mathfrak{R}}\right)^{2} d r .
\end{gathered}
$$

Hence, we obtain the initial energy distribution of the substance:

$$
\frac{d m}{d \epsilon}=\left(\frac{3 \Re}{G}\right) \sqrt{3+\frac{2 \epsilon}{\Phi}} ; \quad \epsilon \in\left[-\frac{3}{2} \Phi ;-\Phi\right],
$$

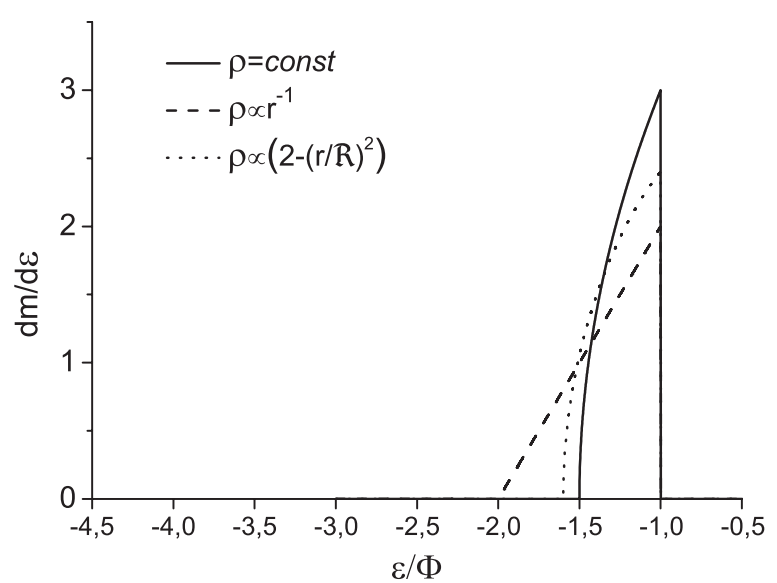

Figure 1. Energy spectra $d m / d \epsilon$ for various forms of initial perturbations. Apparently, there are no particles with $\epsilon>-\Phi$.

where $\Phi \equiv\left(G m / R_{\text {vir }}\right) \simeq(G m / \Re)$. Performing similar calculations, we obtain for the case of initial density perturbation $\rho \propto r^{-1}$

$$
\frac{d m}{d \epsilon}=\frac{2 m}{\Phi}\left(2+\frac{\epsilon}{\Phi}\right), \quad \epsilon \in[-2 \Phi ;-\Phi] .
$$

As one can see in Figure 1, distributions (Equations (6) and (7)) do not differ strongly: all the particles lie in a respectively narrow energy range $[-2 \Phi ;-\Phi]$, and the majority of the particles have energies close to $-\Phi$. Moreover, the initial energy spectrum of any real (smooth) density perturbation lies between Equations (6) and (7): the perturbation collapses when $\delta \rho / \rho=$ $\beta \simeq 1$, and $\beta$ weakly depends on its shape. Consequently, if the initial shape of $\delta \rho(r)$ is a smooth function, distributions of $\phi$ and $d m$ differ from Equations (4) and (5) by only a multiplier of the order of two. As a result, distribution $d m / d \epsilon$ does not differ very significantly from the uniform one (Equation (6)). As an illustration, we also consider profile $\rho \propto\left(2-(r / \mathfrak{R})^{2}\right)$, which approximates well real initial perturbations. The corresponding energy distribution, represented in Figure 1, turns out to be quite close to Equation (6).

It seems reasonable to say that the initial energy distribution is similar to Equation (6) under any reasonable choice of the shape of the initial perturbations. If we divide a spherically symmetric initial distribution on radial layers of thickness $d r, \epsilon$ is constant within the layer, and the volume of the layer grows as $(r / \mathfrak{R})^{2}$. As a result, the particles with $\epsilon \simeq-\Phi$ (which corresponds to $r=\mathfrak{R}$ ) dominate in the initial energy spectrum even in the case of a quite steep profile $\left(\delta \rho \propto r^{-1}\right)$. A strong departure from Equation (6) will take place only if the initial profile is very steep (for instance, $\delta \rho \propto r^{-3}$ ). On the other hand, such profiles are hardly suitable for describing the initial perturbations of DM.

Thus, for a large variety of initial conditions, the initial energy distribution of the particles is similar to Equation (6), at least in the following sense: the majority of the particles have energy close to $-\Phi$, and their energy distribution is quite narrow. In the case of distribution (6), for instance, for all particles, $\epsilon \in[-(3 / 2) \Phi ;-\Phi]$. This conclusion is also valid for warm DM. Indeed, the smearing of the energy spectrum with respect to the CDM case is of the order of the temperature of DM at the moment of structure collapse. For the structure to be able to collapse, the temperature should be much lower than $\Phi$.

In view of our principle assumption that the energy distribution of the particles changes moderately with respect to the 
initial one, the similarity between the initial distributions leads to a similarity among the particle energy spectra of the relaxed halo. But how can the system evolve at all if the energies of the bulk of the particles remain almost the same? The mechanism is as follows: the particles oscillate in the potential well. Initially, they oscillate cophasally. Then, even if the energies of the particles remain constant, their phases diverge. Typically, the period sharply depends on $\epsilon$ for the majority of potential wells. However, even if the energies of the particles precisely coincide, their phases can diverge as a result of a small nonsphericalness, perturbations from the nearby structures, etc. Finally, the phases of the oscillations are mixed thoroughly, and each phase becomes equiprobable. This is exactly the situation that corresponds to the stationary halo. It is important to underline that the phase mixing goes on continuously, in contrast to the energy exchange, which is effective only during the first short stage of violent relaxation.

Finally, the halo collapse forms a stationary potential well $\phi(r)$, where $\phi$ does not depend on time. Each particle can be characterized by its specific angular momentum $\mu=|[\boldsymbol{v} \times \boldsymbol{r}]|$ and the maximum radius $r_{0}$ from the center that the particle can reach. The radius $r_{0}$ is bound on a one-to-one basis to the specific particle energy by the evident relationship $\epsilon=\phi\left(r_{0}\right)+\mu^{2} /\left(2 r_{0}^{2}\right)$. We may consider the halo particle distribution function over $r_{0}$ :

$$
d m=f\left(r_{0}\right) d r_{0}
$$

If the energy evolution of the halo is moderate, $f\left(r_{0}\right)$ has a very peculiar appearance. Indeed, the potential well of the collapsed halo is much deeper than the initial one. An initial perturbation had $\left.\left.k \equiv \mid\left(\phi\left(R_{\text {vir }}\right)-\phi(0)\right) / \phi\left(R_{\text {vir }}\right)-\phi(\infty)\right)\right) \mid \leqslant 1$, as we could see from Equations (6) and (7), while a formed galactic halo always has $k \gg 1$. For instance, a NFW halo has $k \simeq c_{\text {vir }}$ (Navarro et al. 1997; Baushev 2012). Accepting $M_{\mathrm{vir}}=10^{12} M_{\odot}, R_{\mathrm{vir}}=250 \mathrm{kpc}$ for the Milky Way (Klypin et al. 2002), we obtain $-\Phi=-\phi\left(R_{\text {vir }}\right) \simeq\left(130 \mathrm{~km} \mathrm{~s}^{-1}\right)^{2}$. Meanwhile, the escape speed near the solar system undoubtedly exceeds $525 \mathrm{~km} \mathrm{~s}^{-1}$ (Carney \& Latham 1987) and may, in principle, be much larger $\left(650 \mathrm{~km} \mathrm{~s}^{-1}\right.$ or even higher; Marochnik \& Suchkov 1984; Binney \& Tremaine 2008). This means that $k \geqslant 10$ for the Milky Way and should be even higher for less massive systems. The specific energies of the particles bound in such a well may, in principle, lie between $\epsilon=-\Phi\left(r_{0} \simeq R_{\mathrm{vir}}\right)$ and $\epsilon=-k \Phi \simeq-c_{\text {vir }} \Phi\left(r_{0}=0\right)$. However, if we accept principle assumption (3), the real energy range occupied by the particles is narrower. Indeed, as we can see, the particles are strongly concentrated toward $\epsilon=-\Phi$ in any reasonable initial perturbation, and if we consider profile $\rho \propto\left(2-(r / \mathfrak{R})^{2}\right)$ as a good approximation of real initial perturbations, we see that the energies of all the particles fall in $\epsilon \in[-\Phi ;-1.6 \Phi]$ (see Figure 1). If assumption (3) is true, it suggests that the majority of the particles still concentrate near $\epsilon=-\Phi$ : the energy exchange of the particles during the relaxation is more or less a stochastic process, while the total energy of the system should be conserved. Thus, the narrowness of the initial energy distribution, the relative smallness of the energy evolution, and the fact that the final potential well is much deeper than the initial one together result in a crowding of the particle apocenter distances $r_{0}$ near $R_{\text {vir }}$.

However, a consequence of assumption (3) much more important for us is that the halo contains almost no particles with energies less than $\epsilon=-1.6 \Phi \times c_{\text {vir }} / 3 \simeq-\left(c_{\mathrm{vir}} / 2\right) \Phi$. The radius $r_{0}$, corresponding to $\epsilon=-\left(c_{\mathrm{vir}} / 2\right) \Phi$, depends on the density profile. However, it a fortiori exceeds ${ }^{1} 2 R_{\mathrm{vir}} / c_{\mathrm{vir}}$, which corresponds to the gravitational field of a point mass $M_{\text {vir }}$; for a highly concentrated NFW halo $\epsilon=-\left(c_{\text {vir }} / 2\right) \Phi$ corresponds to $r_{0} \simeq 2.5 R_{\mathrm{vir}} / c_{\mathrm{vir}}$. Since condition (3) states that a halo contains only a few particles with $\epsilon<-\left(c_{\text {vir }} / 2\right) \Phi$, the halo should contain only a few particles with $r_{0} \in\left[0 ; 2 R_{\text {vir }} / c_{\text {vir }}\right]$. Actually, the number of particles with $r_{0} \sim 2 R_{\mathrm{vir}} / c_{\mathrm{vir}}$ should also be relatively small since the initial distribution $\rho \propto$ $\left(2-(r / \mathfrak{R})^{2}\right)$ contains only a few particles with $\epsilon \simeq-1.6 \Phi$. We may conclude that the distribution function $f\left(r_{0}\right)$ has a steep maximum near $r_{0} \sim R_{\mathrm{vir}}$ and that it is almost equal to zero when $r_{0} \sim(2-3) R_{\mathrm{vir}} / c_{\mathrm{vir}}$.

The latter property is the most important for us. Although the fraction of particles with $r_{0} \leqslant(2-3) R_{\mathrm{vir}} / c_{\mathrm{vir}}$ is small, the region $r \leqslant(2-3) R_{\text {vir }} / c_{\text {vir }}$ is quite large (40-50 kpc for our Galaxy) and contains a very significant fraction of the galaxy mass, which means that the dominant contribution to the DM profile in the halo center is given by particles that come from the outside, i.e., that have apocenter distances $r_{0}$ significantly larger than the radius of the region under discussion. Hereafter we will consider an even smaller area around the halo center, $r \leqslant R_{\text {vir }} / c_{\text {vir }}$, where we thus have $r \ll r_{0}$ for almost all the particles. On the other hand, this area is still quite large $\left(R_{\mathrm{vir}} / c_{\mathrm{vir}} \simeq 20 \mathrm{kpc}\right.$ for the Milky Way). Moreover, $r=R_{\mathrm{vir}} / c_{\mathrm{vir}}$ corresponds to the radius where a NFW halo has $d \log \rho(r) / d \log r=-2$; consequently, we may expect that if a halo has a core, its radius is smaller than the radius of the area under consideration.

As we will see, if the central profile is created by particles with $r \ll r_{0}$, the DM density profile is quite universal, does not depend on the detailed properties of the distribution $f\left(r_{0}\right)$, and is close to the Einasto profile with $n \simeq 0.5$.

\section{CALCULATIONS}

We can easily find the density distribution in the center of the halo, created by the particles coming to this region from the outside, using the method offered in Baushev (2013a). Let us single out particles with a certain $r_{0}$. We denote their total mass by $m$. $N$-body simulations (Hansen et al. 2006; Kuhlen et al. 2010) suggest that the distribution over tangential velocities is close to but not exactly Gaussian. According to these results, we assume for simplicity that the specific angular momentum $\mu \equiv[\boldsymbol{v} \times \boldsymbol{r}]$ of the particles has a Gaussian distribution,

$$
d m=m \frac{2 \mu}{\alpha^{2}} \exp \left(-\frac{\mu^{2}}{\alpha^{2}}\right) d \mu .
$$

Here $\alpha \equiv \alpha\left(r_{0}\right)$ is the width of the distribution; generally speaking, $\alpha$ is a function of $r_{0}$. As we can see, the majority of the particles have $r_{0} \sim R_{\text {vir }}$, so only those with small $\mu$ can penetrate into the area that interests us, $r \sim R_{\text {vir }} / c_{\text {vir. }}$. Hence, the distribution in the halo center is mainly determined by the behavior of Equation (9) when $\mu \rightarrow 0$. Thus, our calculation is not very sensitive to the assumption of a Gaussian distribution: any other distribution with the same behavior at $\mu \simeq 0$ would give a similar result.

A particle moving in gravitational field $\phi(r)$ has two integrals of motion: $\mu$ and $\epsilon=v_{r}^{2} / 2+\mu^{2} / 2 r^{2}+\phi$. The radial velocity of

\footnotetext{
1 Here we neglect the part of the particle energy associated with the angular momentum $\mu^{2} / 2 r^{2}$. $N$-body simulations show that the orbits of the majority of the particles are rather prolate (Diemand \& Kuhlen 2008), and in this case the influence of the angular momentum on $r_{0}$ is negligible. However, even in the worst case of a circular orbit, $r_{0}$ of a particle cannot be more than two times smaller than the one for a radially moving particle with the same energy.
} 
the particle is equal to

$$
v_{r}=\sqrt{2\left(\phi\left(r_{0}\right)-\phi(r)\right)-\mu^{2}\left(\frac{1}{r^{2}}-\frac{1}{r_{0}^{2}}\right)} .
$$

We introduce the maximum angular momentum for a particle to be able to reach radius $r$,

$$
\mu_{\max }^{2}=2\left(\phi\left(r_{0}\right)-\phi(r)\right)\left(\frac{1}{r^{2}}-\frac{1}{r_{0}^{2}}\right)^{-1} .
$$

Then Equation (10) may be rewritten as

$$
v_{r}=\frac{\sqrt{r_{0}^{2}-r^{2}}}{r r_{0}} \sqrt{\mu_{\max }^{2}-\mu^{2}} .
$$

A particle having maximal radius $r_{0}$ and minimal radius $r_{\min }$ contributes to the halo density in the interval $\left[r_{\min }, r_{0}\right]$. The contribution of a single particle of mass $m$ in an interval $d r$ is proportional to the time the particle passes in this interval (Baushev 2011):

$$
\frac{d m}{m}=\frac{d t}{T}=\frac{d r}{v_{r} T} .
$$

Here $T$ is the half period of the particle, i.e., the time it takes for the particle to fall from its maximal radius to the minimal one,

$$
T\left(r_{0}, \mu\right)=\int_{r_{\min }}^{r_{0}} \frac{d r}{v_{r}} .
$$

$T\left(r_{0}, \mu\right)$ is, generally speaking, a function of $r_{0}$ and $\mu$.

We can obtain the density distribution from Equation (13), substituting Equation (12) there instead of $v_{r}$ and $4 \pi r^{2} \rho$ instead of $d m$ :

$$
\rho=\frac{m}{4 \pi r^{2} v_{r} T}=\frac{m r_{0}}{4 \pi r T \sqrt{r_{0}^{2}-r^{2}} \sqrt{\mu_{\max }^{2}-\mu^{2}}} .
$$

In order to find the density distribution produced by all the particles of the halo with a certain $r_{0}$, we should integrate Equation (15) over distribution (9):

$$
\rho=\frac{m r_{0}}{4 \pi r \sqrt{r_{0}^{2}-r^{2}}} \int_{0}^{\mu_{\max }} \frac{2 \mu \exp \left(-\mu^{2} / \alpha^{2}\right)}{\alpha^{2} T\left(r_{0}, \mu\right) \sqrt{\mu_{\max }^{2}-\mu^{2}}} d \mu .
$$

This is the exact solution; we can significantly simplify it, however, if we take into account that $T\left(r_{0}, \mu\right)$ is, in general, a very weak function of $\mu$, especially for small $\mu$, since $T\left(r_{0}, \mu\right)$ has an extremum at $\mu=0$. As we see in Figure 2, $T(\mu)$ differs from $T(0)$ by no more than $25 \%$ for any reasonable potential if $\mu \leqslant 0.5 \mu_{\text {circ }}$, where $\mu_{\text {circ }}$ is the angular momentum corresponding to the circular orbit. Only particles with small $\mu$ can reach the central region under consideration; consequently, we may approximate $T$ by $T\left(r_{0}, \mu\right) \simeq T\left(r_{0}\right) \equiv T\left(r_{0}, 0\right)$. Then we can take the integral in Equation (16):

$$
\rho=\frac{m r_{0}}{2 \pi \alpha T\left(r_{0}\right) r \sqrt{r_{0}^{2}-r^{2}}} D\left(\frac{\mu_{\max }}{\alpha}\right),
$$

where $D$ is the Dawson function $D(x) \equiv e^{-x^{2}} \int_{0}^{x} e^{t^{2}} d t$. Of course, the particles in a real halo have some distribution

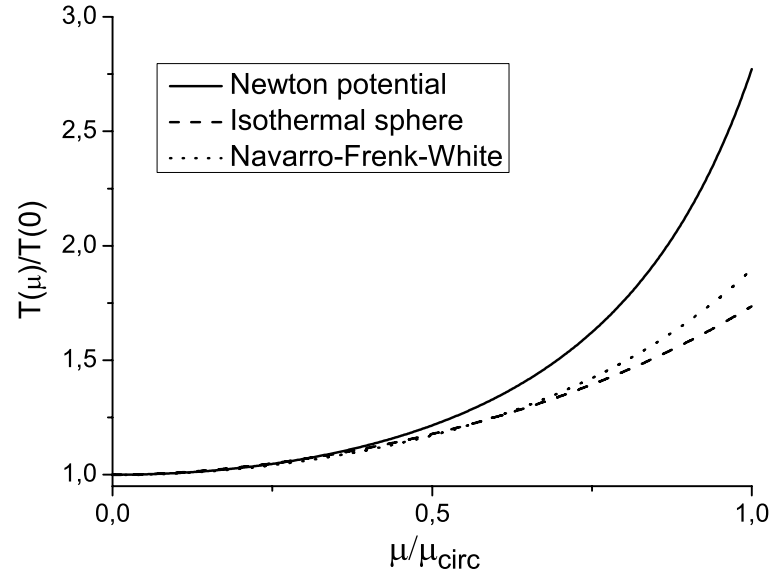

Figure 2. Ratios $T(\mu) / T(0)$ for the Newtonian potential $\phi \propto-1 / r$ (solid line), isothermal sphere $\phi \propto \ln (r)$ (dashed line), and Navarro-Frenk-White profile with $c_{\text {vir }}=5$ (dotted line). The normalization of the potentials is chosen so that the mass inside $r_{0}$ is the same for all three cases. Here $\mu_{\text {circ }}$ corresponds to the circular orbit.

over $r_{0}: d m=f\left(r_{0}\right) d r_{0}$. In order to take it into account, we should substitute $f\left(r_{0}\right) d r_{0}$ instead of $m$ in Equation (17) and integrate the result over $r_{0}$. Moreover, Equation (17) can be significantly simplified in the central part of the halo. First of all, the gravitational potential of the center of the halo, $\phi(0)$, is finite for any reasonable halo profile (otherwise, the annihilation signal produced by the halo would be infinite, being proportional to $\left.\int 4 \pi r^{2} \rho^{2} d r\right)$. Then we may rewrite Equation (11) as

$$
\mu_{\max }^{2}=2 r^{2}\left(\phi\left(r_{0}\right)-\phi(0)\right)\left(1-\frac{\phi(r)-\phi(0)}{\phi\left(r_{0}\right)-\phi(0)}\right) \frac{r_{0}^{2}}{r_{0}^{2}-r^{2}} .
$$

The two last factors may be neglected in the center of the halo, and we can use the approximations $\mu_{\max } \simeq r \sqrt{2\left(\phi\left(r_{0}\right)-\phi(0)\right)}$, $\sqrt{r_{0}^{2}-r^{2}} \simeq r_{0}$. Therefore, the total density distribution in the central part is

$$
\rho=\int_{0}^{\infty} \frac{f\left(r_{0}\right)}{2 \pi \alpha\left(r_{0}\right) T\left(r_{0}\right) r} D\left(r \frac{\sqrt{2\left(\phi\left(r_{0}\right)-\phi(0)\right)}}{\alpha\left(r_{0}\right)}\right) d r_{0} .
$$

We can simplify this equation with the help of the following reasoning. As we can see, $f\left(r_{0}\right)$ is almost equal to zero for small $r_{0}$. Moreover, the halo that is formed is dominated by particles with $r_{0} \sim R_{\text {vir }}$, which means that the main contribution to the integral in Equation (19) is given by the part where $r_{0} \simeq R_{\mathrm{vir}}$, roughly speaking, by $r_{0} \in\left[R_{\mathrm{vir}} / 2 ; R_{\mathrm{vir}}\right]$. As we can see, the function $f\left(r_{0}\right)$ sharply depends on $r_{0}$ in this interval. In contrast, $\alpha\left(r_{0}\right)$ should not change much in the interval $\left[R_{\mathrm{vir}} / 2 ; R_{\mathrm{vir}}\right]: \alpha\left(r_{0}\right)$ is widely believed to be a powerlaw dependence with an index between -1 and 1 (Hansen et al. 2006). Note that $\sqrt{2\left(\phi\left(r_{0}\right)-\phi(0)\right)}$ changes even more slowly: for instance, $\sqrt{\left(\phi\left(R_{\mathrm{vir}}\right)-\phi(0)\right) /\left(\phi\left(R_{\mathrm{vir}} / 2\right)-\phi(0)\right)} \simeq 1.1$ for the NFW profile with $c_{\text {vir }}=15$. Moreover, $D$ is a finite and not very sharp function of its argument. Comparing this with the sharp behavior of $f\left(r_{0}\right)$, we may neglect the weak dependence of the argument of function $D$ in Equation (19) on $r_{0}$ and substitute there some value, averaged over the halo,

$$
r_{c}=\left\langle\frac{\alpha\left(r_{0}\right)}{\sqrt{2\left(\phi\left(r_{0}\right)-\phi(0)\right)}}\right\rangle \simeq \frac{\left\langle\alpha\left(r_{0}\right)\right\rangle}{\sqrt{2|\phi(0)|}} .
$$




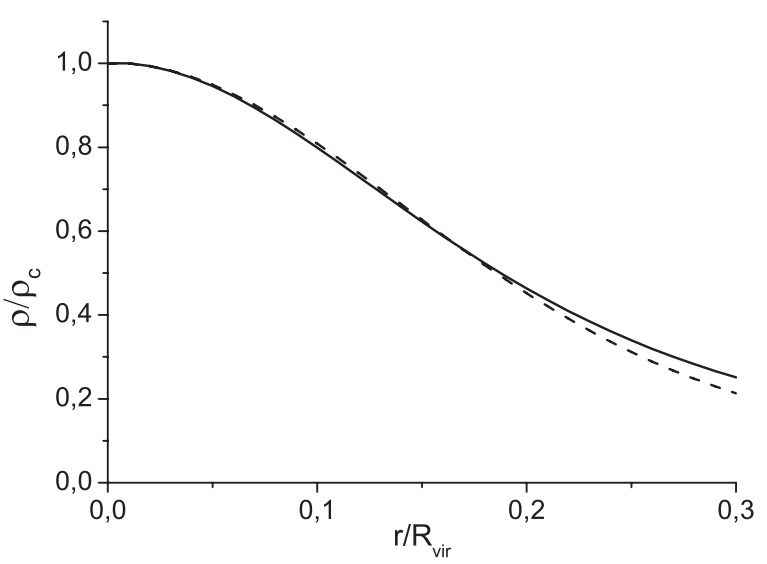

Figure 3. Density profile of the exact solution of Equation (16) for $f\left(r_{0}\right)=$ $M_{\mathrm{vir}} \delta\left(r_{0}-R_{\mathrm{vir}}\right)$ (dashed line) and profile (21) with the same $r_{c} / R_{\mathrm{vir}}$ (solid line). One can see that the departure of approximative Equation (21) from the exact solution is quite small.

Then we can rewrite Equation (19) and obtain the final result:

$$
\rho=\rho_{c} \frac{r_{c}}{r} D\left(\frac{r}{r_{c}}\right), \quad \rho_{c}=\frac{1}{2 \pi r_{c}} \int_{0}^{\infty} \frac{f\left(r_{0}\right) d r_{0}}{\alpha\left(r_{0}\right) T\left(r_{0}\right)} .
$$

Since $D\left(r / r_{c}\right) \simeq r / r_{c}$, when $r / r_{c} \rightarrow 0, \rho_{c}$ is the central density of the halo. As we can see, it is always finite. At the same time, the shape of the density profile depends on only the parameter $r_{c} ; r_{c}$ defines the radius at which a rather shallow central region of the density profile transforms into a much steeper outer part. Therefore, it is actually the core radius of profile (21).

In deriving Equation (21), we used the fact that $r \ll r_{0}$ several times. This raises the following question: for what $r / R_{\text {vir }}$ is Equation (21) still valid? In order to check this, we solved the numerically exact Equation (16) on condition (9) and for the distribution $f\left(r_{0}\right)=M_{\mathrm{vir}} \delta\left(r_{0}-R_{\mathrm{vir}}\right)$. Since our approximation $T\left(r_{0}, \mu\right) \simeq T\left(r_{0}, 0\right)$ is the least acceptable for the particles with a high angular momentum, we used the maximal possible value for $\langle\alpha\rangle=(1 / 4) R_{\mathrm{vir}} \sqrt{G M_{\mathrm{vir}} / R_{\mathrm{vir}}}=$ $(1 / 4) \sqrt{G M_{\text {vir }} R_{\text {vir }}}$. It is apparent that $\langle\alpha\rangle$ cannot substantially exceed this estimation; otherwise, a significant part of the DM would not be gravitationally bound in the halo. The potential $\phi(r)$ appears implicitly in expression (16), and we need to write out the equation

$$
\frac{d \phi}{d r}=\frac{G}{r^{2}} \int_{0}^{r} 4 \pi x^{2} \rho(x) d x
$$

in order to close the task. The exact density profile (dashed line) and profile (21) with the same $r_{c} / R_{\mathrm{vir}}$ (solid line) are represented in Figure 3. We see that Equation (21) approximates well the exact solution up to $r \simeq 0.3 R_{\text {vir }}$. Therefore, Equation (21) can be valid over quite a large area around the halo center.

\section{DISCUSSION}

Figure 4 represents density profile (21) for $r_{c}=R_{\mathrm{vir}} / 20$. For comparison, the Einasto profile with $n=0.5$ and $r_{s}=0.017 R_{\text {vir }}$ is also plotted. As we can see, the profiles are quite similar; that is, Equation (21) has a core and is close to the Einasto profile with $n=0.5$, which, in turn, fits well observational DM profiles of numerous galaxies (Chemin et al. 2011).

There are two more properties of profile (21) that can be observationally checked. First, since $D(x) \simeq x^{-1}$ when

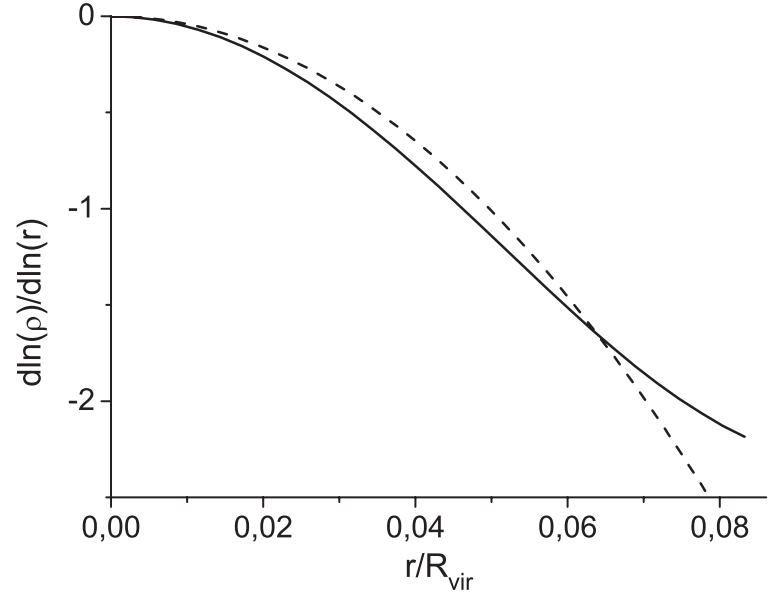

Figure 4. Density profile (21) of the central region of a halo with $r_{c}=R_{\mathrm{vir}} / 20$. The Einasto profile with $n=0.5$ and $r_{s}=0.017 R_{\mathrm{vir}}$ is plotted for comparison (dashed line).

$x \rightarrow \infty$, density distribution (21) behaves as $\rho \propto r^{-2}$ at large distances $\left(r \gtrsim 1.6 r_{c}\right)$. The outer border of the applicability of profile (21) is defined by the size of the area where the distribution $f\left(r_{0}\right)$ is almost equal to zero (see the end of Section 2), i.e., $r \simeq 2 R_{\text {vir }} / c_{\text {vir. }}$. Since $1.6 r_{c}$ can be significantly smaller for real galaxies, we may expect a large area with $\rho \propto r^{-2}$ in the density profile between $r \gtrsim 1.6 r_{c}$ and $r \simeq 2 R_{\text {vir }} / c_{\text {vir }}$. This region is actually observed in the profiles of many galaxies (Sofue \& Rubin 2001). It is the feature that allowed the existence of DM to be proved in 1978 (Rubin et al. 1978). Meanwhile, neither NFW nor the Einasto profile has an explicit region with $\rho \propto r^{-2}$. Of course, the power index $d \log \rho(r) / d \log r$ reaches -2 at some radius. This point is in no way separated, however, and there is no reason to expect a large region with the isothermal profile. Although there could be other reasons for its appearance, such as the baryon influence, the $\rho \propto r^{-2}$ profile persistently occurs in many galaxies with very different properties, which implies a fundamental physical mechanism. Profile (21) gives a simple and natural explanation of this effect.

We may roughly estimate the multiplication of the central density $\rho_{c}$ on the core radius $r_{c}$ (Baushev 2013b). Indeed, if the particles with a certain $r_{0}$ have a characteristic velocity $v$, then $\alpha\left(r_{0}\right) \sim r_{0} v, T\left(r_{0}\right) \sim \tau_{d}=r_{0} / v$, and $\alpha\left(r_{0}\right) T\left(r_{0}\right) \sim r_{0}^{2}$. Substituting this value into Equation (21), we obtain

$$
\rho_{c} r_{c} \propto \int_{0}^{\infty} \frac{f\left(r_{0}\right)}{r_{0}^{2}} d r_{0} .
$$

Now we can follow the same reasoning that we used to transform Equation (19) into Equation (20): $f\left(r_{0}\right)$ is nonvanishing at $r \in\left[2 R_{\mathrm{vir}} / c_{\mathrm{vir}}, R_{\mathrm{vir}}\right]$. In this interval $r_{0}^{2}$ varies much slower than $f\left(r_{0}\right)$, and we may substitute some value $\left\langle r_{0}^{2}\right\rangle$ averaged over the halo for it. Then

$$
\rho_{c} r_{c} \propto \frac{\int_{0}^{\infty} f\left(r_{0}\right) d r_{0}}{\left\langle r_{0}^{2}\right\rangle}=\frac{M_{\mathrm{vir}}}{\left\langle r_{0}^{2}\right\rangle}
$$

As we can see, the majority of the particles have $r_{0} \sim R_{\mathrm{vir}}$, and it is reasonable to assume that $\left\langle r_{0}^{2}\right\rangle \propto R_{\mathrm{vir}}^{2}$. Thus, $\rho_{c} r_{c} \propto M_{\mathrm{vir}} / R_{\mathrm{vir}}^{2}$. It is more convenient to use the average halo density $\varrho$ : $M_{\mathrm{vir}}=(4 / 3) \pi R_{\mathrm{vir}}^{3} \varrho$. We obtain

$$
\rho_{c} r_{c} \propto M_{\mathrm{vir}}^{1 / 3} \varrho^{2 / 3}
$$


Thus, our model predicts that the multiplication of the central halo density on the core radius should be nearly constant for galaxies. Indeed, let us consider halos with masses $10^{8} M_{\odot}$ and $10^{12} M_{\odot}$, which covers almost the whole range of galaxy masses. We denote the quantities related to $10^{8} M_{\odot}$ and $10^{12} M_{\odot}$ halos by subindices 8 and 12 , respectively. $M_{\text {vir }}^{1 / 3}$ increases by a factor of $\simeq 20$ over the whole mass interval under consideration. Moreover, the second multiplier $\varrho^{2 / 3}$ decreases with $M_{\text {vir }}$ since less massive galaxies formed earlier, when density of the universe was higher. Thus, the multiplier variations partially compensate each other. Virial halo density $\varrho$ and $M_{\text {vir }}$ are not one-to-one related. The structures appeared from a Gaussian random field, and objects of the same $M_{\text {vir }}$ may have different $\varrho$. However, presuming that galaxies with $10^{8} M_{\odot}$ and $10^{12} M_{\odot}$ were formed at $z_{8}=10$ and $z_{12}=4$, respectively, we may assume that their density ratio is roughly equal to $\varrho_{8} / \varrho_{12} \simeq\left(\left(z_{8}+1\right) /\left(z_{12}+1\right)\right)^{3} \simeq 8$ (Gorbunov \& Rubakov 2011). Thus, our model (21) predicts that $\rho_{c} r_{c} \propto M_{\mathrm{vir}}^{1 / 3} \varrho^{2 / 3}$ changes only by a factor of five when $M_{\text {vir }}$ runs over the whole range of the galaxy masses. This prediction is also strongly supported by observations. Kormendy \& Freeman (2004) first discovered the constancy of the multiplication of the central halo density in the core radius, and then it was confirmed by several independent observations (see Donato et al. 2009, and references therein). The observations suggest that $\log \left(\rho_{c} r_{c}\right)=1.88 \pm 0.2$ in units of $\log \left(M_{\odot} \mathrm{pc}^{-2}\right)$ for thousands of galaxies with various physical properties.

Thus, the supposition of the moderate energy evolution would suffice to explain three observational properties of galactic halos. Profile (21) is in agreement with observational data for at least some types of galaxies but clearly disagrees with $N$-body simulations and observational data for galaxy clusters (Okabe et al. 2010). This suggests that violent relaxation takes place in galaxy clusters but does not occur in galaxies. There could be various plausible explanations for this: the baryon influence, the warmth of the DM, etc. However, the cause may be much simpler: galaxy clusters have low concentrations $c_{\text {vir }}=3-5$. Condition (3) is very strict for an object with $c_{\text {vir }}=4$ (the energy of the majority of the particles should not change by more than $30 \%$ ) and unsatisfiable for $c_{\text {vir }}<3$. Galaxies have significantly higher $c_{\mathrm{vir}}: c_{\mathrm{vir}}=15-20$ for massive galaxies and is probably even higher for dwarf objects. In order to be violent, the relaxation should change the energies of a significant part of the particles by $30 \%-50 \%$ in the case of galaxy clusters and at least a factor of five in the case of galaxies. Consequently, the relaxation may be violent enough to form cusps in galaxy clusters but moderate in galaxies.

We can now specify the condition of feasibility of our principle assumption: a moderate energy evolution of the system during the halo formation. We are interested in the density profile in the very center of the halo ( $r \sim r_{c}$, where $r_{c}$ is the core radius). We can divide all the particles in this area into two groups: (1) the particles residing in this area (with $r_{0} \lesssim r_{c}$ ) and (2) the outer particles $\left(r_{0} \gg r_{c}\right)$. Both groups give some yield into the density profile of the central region. Our consideration (in particular, the derivation of Equation (21)) shows that the contribution of group 2 is always Einasto-like with a small Einasto index ( $n \simeq 0.5$ ). In order to ascertain that the total density profile of the central part of the halo is close to Equation (21), we only need the contribution of group 1 to be small. This requirement is met automatically if the energy exchange between DM particles is moderate in the sense of Equation (3).

We would like to summarize the main conclusions of this article. First, if the halo relaxation is moderate, the density profile in the central part of a formed halo is described by Equation (21), i.e., is Einasto-like with a small Einasto index ( $n \simeq 0.5$ ). The profile is quite universal and weakly depends on the shape of the initial perturbation. This is a result of the fact that the potential well of the formed halo is much deeper than that of the initial perturbation. As a result, quite a large area builds up in the center of the halo; the particles do not reside in the center but come there from the outside: their apoapses lie outside of the area. In order for the orbit of a particle to lie entirely within the area, the particle energy should be dramatically decreased with respect to the initial value. Such a process is always hampered in a dissipationless system, and hence, the particles, which come to the central area from the outside, dominate there and form Einasto-like universal density profile (21). The shape of the profile is totally determined by a single parameter, $r_{c}$ (Equation (20)), which can be considered the core radius. The model gives a natural explanation for the large areas with the nearly isothermal profile $\rho \propto r^{-2}$ routinely observed in spiral galaxies. Moreover, it predicts the constancy of the multiplication of the central density $\rho_{c}$ in the core radius $r_{c}$ of DM halos, in good agreement with astronomical data.

Financial support by Bundesministerium für Bildung und Forschung through DESY-PT, grant 05A11IPA, is gratefully acknowledged. BMBF assumes no responsibility for the contents of this publication. We acknowledge support by the Helmholtz Alliance for Astroparticle Physics (HAP) funded by the Initiative and Networking Fund of the Helmholtz Association.

\section{REFERENCES}

Baushev, A. N. 2011, MNRAS, 417, L83

Baushev, A. N. 2012, MNRAS, 420, 590

Baushev, A. N. 2013a, ApJ, 771, 117

Baushev, A. N. 2013b, arXiv:1309.5162

Baushev, A. N. 2013c, arXiv:1312.0314

Binney, J., \& Tremaine, S. 2008, Galactic Dynamics (2nd ed.; Princeton, NJ: Princeton Univ. Press)

Carney, B. W., \& Latham, D. W. 1987, in IAU Symp. 117, Dark Matter in the Universe, ed. J. Kormendy \& G. R. Knapp (Dordrecht: Reidel), 39

Chemin, L., de Blok, W. J. G., \& Mamon, G. A. 2011, AJ, 142, 109

de Blok, W. J. G., \& Bosma, A. 2002, A\&A, 385, 816

de Blok, W. J. G., McGaugh, S. S., \& Rubin, V. C. 2001, AJ, 122, 2396

Diemand, J., \& Kuhlen, M. 2008, ApJL, 680, L25

Diemand, J., Kuhlen, M., \& Madau, P. 2007, ApJ, 667, 859

Diemand, J., Kuhlen, M., Madau, P., et al. 2008, Natur, 454, 735

Diemand, J., Madau, P., \& Moore, B. 2005, MNRAS, 364, 367

Donato, F., Gentile, G., Salucci, P., et al. 2009, MNRAS, 397, 1169

Einasto, J. 1965, TrAlm, 51, 87

Evans, N. W., \& Collett, J. L. 1997, ApJL, 480, L103

Gao, L., Navarro, J. F., Cole, S., et al. 2008, MNRAS, 387, 536

Garrison-Kimmel, S., Rocha, M., Boylan-Kolchin, M., Bullock, J. S., \& Lally, J. 2013, MNRAS, 433, 3539

Gentile, G., Salucci, P., Klein, U., \& Granato, G. L. 2007, MNRAS, 375, 199

Gorbunov, D. S., \& Rubakov, V. A. 2011, Introduction to the Theory of the Early Universe (Singapore: World Scientific)

Governato, F., Zolotov, A., Pontzen, A., et al. 2012, MNRAS, 422, 1231

Hansen, S. H., Moore, B., Zemp, M., \& Stadel, J. 2006, JCAP, 01, 014

Hayashi, E., Navarro, J. F., Taylor, J. E., Stadel, J., \& Quinn, T. 2003, ApJ, 584,541

Jaffe, W. 1983, MNRAS, 202, 995

Klypin, A., Prada, F., Yepes, G., Hess, S., \& Gottlober, S. 2013, arXiv:1310.3740 Klypin, A., Zhao, H., \& Somerville, R. S. 2002, ApJ, 573, 597

Kormendy, J., \& Freeman, K. C. 2004, in IAU Symp. 220, Dark Matter in Galaxies, ed. S. Ryder, D. Pisano, M. Walker, \& K. Freeman (San Francisco, CA: ASP), 377

Kuhlen, M., Weiner, N., Diemand, J., et al. 2010, JCAP, 02, 030

Landau, L. D., \& Lifshitz, E. M. 1980, Course of Theoretical Physics, Statistical Physics. Pt.1, Pt.2 (Oxford: Pergamon)

Lynden-Bell, D. 1967, MNRAS, 136, 101 
Marchesini, D., D’Onghia, E., Chincarini, G., et al. 2002, ApJ, 575, 801 Marochnik, L. S., \& Suchkov, A. A. 1984, The Galaxy (Moscow: Izdatel'stvo Nauka)

Moore, B., Lake, G., \& Katz, N. 1998, ApJ, 495, 139

Navarro, J. F., Frenk, C. S., \& White, S. D. M. 1997, ApJ, 490, 493

Navarro, J. F., Ludlow, A., Springel, V., et al. 2010, MNRAS, 402, 21

Oh, S.-H., de Blok, W. J. G., Brinks, E., Walter, F., \& Kennicutt, R. C., Jr. 2011, AJ, 141, 193

Okabe, N., Zhang, Y.-Y., Finoguenov, A., et al. 2010, ApJ, 721, 875

Osipkov, L. P. 1979, PAZh, 5, 77

Planck Collaboration, Ade, P. A. R., Aghanim, N., et al. 2013, arXiv:1303.5076

Pontzen, A., \& Governato, F. 2012, MNRAS, 421, 3464

Pontzen, A., \& Governato, F. 2013, MNRAS, 430, 121
Pontzen, A., \& Governato, F. 2014, Natur, 506, 171

Power, C., Navarro, J. F., Jenkins, A., et al. 2003, MNRAS, 338, 14

Quinlan, G. D. 1996, NewA, 1, 255

Rubin, V. C., Thonnard, N., \& Ford, W. K., Jr. 1978, ApJL, 225, L107

Sofue, Y., \& Rubin, V. 2001, ARA\&A, 39, 137

Stadel, J., Potter, D., Moore, B., et al. 2009, MNRAS, 398, L21

Stiavelli, M., \& Bertin, G. 1987, MNRAS, 229, 61

Syer, D., \& White, S. D. M. 1998, MNRAS, 293, 337

Tremaine, S., Henon, M., \& Lynden-Bell, D. 1986, MNRAS, 219, 285

Wang, J., Frenk, C. S., Navarro, J. F., Gao, L., \& Sawala, T. 2012, MNRAS, 424, 2715

Weinberg, D. H., Bullock, J. S., Governato, F., Kuzio de Naray, R., \& Peter, A. H. G. 2013, arXiv:1306.0913 